\title{
El papel del consumo en la economía de plataformas: el vínculo oculto
}

\author{
The role of consumption in the platform economy: the hidden \\ link
}

\author{
Luis Enrique Alonso \\ Universidad Autónoma de Madrid, España \\ luis.alonso@uam.es
}

Carlos J. Fernández Rodríguez

Universidad Autónoma de Madrid, España

carlos.fernandez@uam.es

Recibido / Received:21/03/2021

Aceptado / Accepted: 18/04/2021

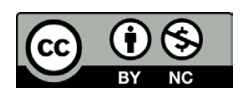

\section{RESUMEN}

La economía de las plataformas o gig economy, además de suponer una auténtica revolución en los mercados, está generando un impacto muy controvertido sobre las condiciones de empleo en muchos sectores, con la proliferación de nuevos trabajos precarios. Sin embargo, no podemos entender esta metamorfosis del trabajo prestando atención únicamente a la esfera de la producción y las estrategias de gestión de las empresas, sino que debemos fijar nuestra mirada en otros aspectos. Uno de ellos, esencial pero generalmente menos explorado en los análisis sobre esta nueva economía, es el del consumo. No hay gig economy sin la emergencia de formas de consumo y estilos de vida muy concretos, vinculados a la digitalización y el uso extendido de los algoritmos como nueva estrategia de segmentación de mercados. En esta contribución, nuestro objetivo es el de ofrecer una reflexión sobre la importancia que el espacio del consumo tiene en la construcción de este modelo económico de las plataformas, discutiendo sus implicaciones y mostrando su vínculo, muchas veces oculto, con el tipo de empleo que se genera.

Palabras clave: plataformas, economía gig, consumo, internet, algoritmo.

\section{ABSTRACT}

The platform economy or gig economy not only represents a real market revolution. It is also generating a highly controversial impact on employment conditions in many economic 
sectors, with the proliferation of new precarious jobs. However, we cannot understand this metamorphosis of work by only paying attention to the organization of production and management strategies: we must explore other aspects. One of them, critical but generally overlooked, is that of consumption. There is no gig economy without the emergence of very specific forms of consumption and lifestyles, linked to digitization and the widespread use of algorithms as a new market segmentation strategy. In this contribution, our aim is to reflect upon the role that consumption plays in building this platform economy model, discussing its implications as well as showing the often hidden link between the realms of consumption and work.

Keywords: platforms, gig economy, consumption, internet, algorithm.

\section{INTRODUCCIÓN}

El ascenso de la denominada economía de plataformas es una de las transformaciones más relevantes que ha experimentado el capitalismo contemporáneo en los últimos tiempos, visto por algunos académicos incluso como una suerte de tercera revolución industrial (Parguel, Lunardo y Benoit-Moureau, 2017). Es el resultado de la consolidación de un capitalismo informacional en el que la información ha adquirido un peso sin precedentes en el modo en que influye, afecta y modela nuestros estilos de vida (Lash, 2007). La idea de economía de plataformas en sí es un concepto genérico, comúnmente utilizado junto con otros también muy populares (gig economy, economía colaborativa, etc.) que agrupa, en realidad, numerosas prácticas mercantiles y no mercantiles incrustadas en los soportes tecnológicos proporcionados por el desarrollo de la llamada web 2.0 (Beer, 2009; Acquier, Daudigeos y Pinkse, 2017). En todo caso, su manifestación más característica ha sido la del dominio comercial de grandes compañías vinculadas al desarrollo de aplicaciones que conectan empresas y consumidores en internet. La hegemonía de gigantes empresariales como Amazon o Alibaba en el comercio electrónico internacional, así como nuevos modelos de negocio, bien inspirados en la denominada economía colaborativa (Uber, AirBnB) o resultado de la innovación en el terreno de las apps tecnológicas (el sector del delivery), han supuesto una verdadera revolución en numerosos mercados, introduciendo prácticas novedosas en la naturaleza y organización del trabajo y en la relación establecida a lo largo de las últimas décadas entre las esferas del trabajo y el consumo (Alonso, Fernández Rodríguez e Ibáñez Rojo, 2020).

En la actualidad, cuando nos centramos en el análisis de esta economía de las plataformas, las ciencias sociales están prestando especial atención a los drásticos cambios que están teniendo lugar en el terreno laboral, una vez que la gig economy está implicando la consolidación de nuevos empleos con condiciones laborales marcadas por la precariedad y la incertidumbre en su estatus legal (¿son empleados o autónomos?), como es el caso de los riders, conductores de transporte privado y otros empleos (ver Fleming, 2017; Howcroft y Bergvall-Kåreborn, 2019; Aroles, Mitev y de Vaujany, 2019; Álvarez Hernández y Pérez Zapata, 2020; Revilla y Blázquez, 2021). Sin embargo, no podemos entender esta metamorfosis del trabajo centrándonos únicamente en la esfera de la producción y las estrategias de gestión de las empresas, sino que debemos fijar nuestra mirada en otros aspectos. Uno de ellos, esencial pero generalmente menos explorado en los análisis sobre esta nueva economía, es el del consumo. Y es que el proceso de precarización y desestabilización radical de las relaciones salariales instauradas por la gig economy parece directamente vinculado con la emergencia de formas de consumo y estilos de vida concretos, establecidos por las estrategias comerciales asociadas al modelo de negocio de la compra telemática y por los procesos de distribución diseñados por las plataformas digitales de venta y reparto. 
Aunque algunas investigaciones han descrito los impactos que esta nueva economía de las plataformas está teniendo sobre ciertas prácticas del consumo ( $p$. e. Schor y Attwood-Charles, 2017; Frick et al, 2020), explorar las transformaciones en el espacio del imaginario del consumo es un proyecto que debe ser profundizado. Además, la actual pandemia ha reforzado y consagrado definitivamente, hasta naturalizarlo y convertirlo en ideológicamente hegemónico, un modelo de consumo digital que ha supuesto reconstruir a fondo las formas de socialización e interacción asociadas al hecho adquisitivo mismo, reconstruyendo sus tiempos y espacios, y con ello reorganizando todas las formas de poder que se articulan en torno a la sociedad de consumo como forma de constitución del vínculo social. En nuestra contribución a este debate, queremos explorar el significado del consumo en un nuevo contexto marcado por la proliferación de algoritmos como herramienta poderosísima de segmentación de los mercados, para después describir el, a nuestro juicio, controvertido modelo de consumidor solitario que termina emergiendo a raíz de estas condiciones, y discutir sus implicaciones. El objetivo de nuestro trabajo es, así, el de realzar la importancia del análisis de la cultura de consumo y los estilos de vida a la hora de desentrañar los desafíos y problemáticas que están surgiendo ante la paulatina consolidación de los modelos de negocio online.

\section{CONSUMIR EN LA ERA DEL ALGORITMO}

El consumidor de los productos de las grandes plataformas comerciales en internet no sólo efectúa una práctica económica en un ámbito diferente al mercado presencial: construye y reconstruye su identidad en un espacio dominado por condicionamientos empresariales directos, que le ofrecen un espejo electrónico distorsionado de su actividad expresamente creado para garantizar la máxima rentabilización mercantil de todas las acciones que realiza a través de las mediaciones informáticas. Este consumidor de y en internet es un consumidor activado a partir de la constitución de un yo informático (Tolentino, 2019) que ofrece sus datos, se adhiere a todo tipo de redes, muestra sus imágenes ideales -su yo ideal en el sentido psicoanalítico del término- y sigue, admira y/o envidia a imágenes de otros -su ideal del yo en los mismos términos psicoanalíticosprocesadas en el mismo espejo comercial de las redes y las plataformas. El consumidor fraguado por la economía de las plataformas no es el consumidor opulento y pasivo de la edad de oro de la sociedad de consumo moderna (ya clásica), que accedía al consumo de masas como forma normalizada y generalizada de conquistar un mundo de bienestar y confort; ni siquiera es el consumidor segmentado y tribalizado de la primera ola de la cultura postmoderna. Es un hiperconsumidor fragmentado e individualizado hasta el solipsismo (Lipovetsky, 2020, p. 254 y ss.), construido sobre el imaginario de su soberanía y superioridad tecnológica, y presentado como soporte transhumano donde se instalan y facilitan todos los procesos de invisibilización y subordinación del mundo del trabajo.

Es relevante comprender la estrategia de la economía de las plataformas para seducir a ese consumidor individualizado, y que va más allá de algunas ventajas evidentes que el canal online aporta al consumidor medio, como que el catálogo de productos y servicios es más amplio, que permite la comparación de productos, o que es más cómodo (y también, que muchas pequeñas empresas que han conseguido visibilidad y espacio para sus negocios gracias a las plataformas). En primer lugar, es imprescindible destacar que las empresas que operan en esta economía forman en su gran mayoría, irónicamente, monopolios de facto o a lo sumo oligopolios, presentándose en muchos casos no como empresas reales sino como meros intermediarios entre los consumidores y los oferentes de productos y servicios. Estos suelen estar basados en servicios a demanda donde las plataformas cuentan con la capacidad de explotar "asimetrías calculadoras" que sitúan a la plataforma en una posición de poder frente al resto de los participantes en la transacción (Shapiro, 2020). Tanto esa labor de intermediación como la gestión del catálogo de lo ofrecido se va a realizar mediante el uso de algoritmos, programaciones matemáticas que ponderan perfiles de consumidor y patrones de consumo a partir de la 
extraordinaria agregación de datos generados por la huella digital de los consumidores y que, más que probablemente, estén cambiando los hábitos de consumo para siempre. $Y$ en cierto sentido no puede ser de otra manera: el aprovechamiento de todos esos datos es, desde la mirada y lógica de los mercados, un imperativo institucional que permite clasificar a los consumidores y ofrecen precios más competitivos, permitiendo un funcionamiento más eficaz del mercado (Fourcade y Healy, 2017).

El algoritmo es, así, uno de los parámetros fundamentales en la construcción de la cultura contemporánea (Striphas, 2015; Seaver, 2017). Forman parte esencial de los procesos de organización del trabajo y la comunicación en el espacio de la web 2.0, sofisticando tanto las estrategias de personalización del servicio al consumidor como las del control del trabajo gracias a los datos explotados por la tecnología de las plataformas (críticas, valoraciones, velocidad de entrega, historial de consulta o de consumo) (Rosenblat y Stark, 2016; Wood et al, 2019). Ello permite una curiosa combinación entre flexibilidad en el trabajo y control del mismo, pues son los datos recogidos a posteriori los que permiten una reasignación de las tareas y la modificación del flujo de la producción. Para el consumidor, es posible que el algoritmo pudiese suponer una solución efectiva al problema de la abundancia en términos de oferta: así lo señala Wright (2015: 160), si bien también indica que evidentemente el algoritmo no opera de forma neutral, sino que es una construcción humana que va a ceñirse a los intereses y objetivos de aquellos que los han programado. Abrir su caja negra es, de hecho, uno de los desafíos más importantes que tiene la investigación social, una vez que la mayor parte de ellos se encuentran en manos privadas y protegidos por el secreto industrial. El objetivo del algoritmo es el de establecer recomendaciones, no solamente de posibles oferentes de servicios (el conductor que necesitamos, el piso que vamos a alquilar) sino también, de los productos que nos puedan interesar o incluso de las relaciones afectivas que podemos tener con otros (Cohn, 2019). Evidentemente, el algoritmo pasará a ser central en el campo del consumo, una vez que la progresiva digitalización y la extraordinaria cantidad de información disponible lo convertirá en la estrategia más efectiva para acceder a unos perfiles de consumidores a los que dirigirse.

La economías de plataformas ha creado, de este modo, un tipo de regulación, previsión y determinación algorítmica de los comportamientos de los consumidores (Tegmark, 2018), donde se combinan producción, distribución y consumo en una escala y mediante un tratamiento de datos (en el que resalta el famoso Big Data) que sobrepasa con mucho las lógicas normalizadas de los mercados tradicionales de masas -o los ajustes finos de la segmentación postfordista-, en cuanto a la manera en la que se conforma la demanda, se organiza la oferta y se distribuyen los productos, funcionando como un todo integrado informáticamente donde el control del consumidor máximamente individualizado es capaz de integrarse en modelos anonimizados de gestión matemática de escala literalmente global (Fin, 2018; Sandin, 2016). La compra mediante un clic acaba movilizado formas económicas que van desde el algoritmo de la determinación de la demanda -almacenando datos, proponiendo todo tipo de productos materiales e inmateriales personalizados en las pantallas, acumulando favoritos o creando sistemas de influencia, pues el algoritmo se presenta como el garante objetivo de dichos procesos (ver Gillespie, 2014) - hasta la utilización más descarnada del trabajo sin derechos, la subcontratación de servicios degradados y a bajo precio o la utilización de formas de actividad laboral en sus límites de carga física y mental. Y esto no es el resultado de la lógica del algoritmo o un mero equilibrio de precios en el mercado sino, más bien, el resultado de asimetrías en la capacidad de fijar unos precios justos, derivados del control ejercido desde las plataformas de la información disponible (Shapiro, 2020). En estos sistemas gigantes de gestión de la información comercial se mezclan, además sin solución de continuidad, la alta tecnología con unos usos laborales que, en muchos casos, tiene claros aspectos prefordistas (Ravanelle, 2019) pese a que se recurra a una tecnología de control del mismo con ciertas resonancias tayloristas (Crouch, 2019).

En la economía de la plataforma, el canal organiza los trabajos de producción y distribución imponiendo condiciones de utilización que garantizan un máximo beneficio 
al intermediario. Así, las partes de las cadenas de valor más rentables se establecen a partir de la desposesión de control, derechos o márgenes a todos los demás participantes y esto hace de la gig economy un juego de dominaciones, donde los superbeneficios se obtienen de desarticular los poderes clásicos de la producción material. Se arrincona de este modo la distribución comercial clásica -grande y pequeña- mediante una administración de márgenes y precios imposibles de replicar por el comercio tradicional (dada la presencia de costes físicos y de infraestructura mucho más elevados) y, por la organización de un sistema servicio de reparto y entrega rápida a domicilio que implica soluciones logísticas integradas en la economía de la información y su organización algorítmica desde la misma compra. Ello implica el uso intensivo de gran cantidad de trabajo inestable, sub o infra contratado capaz de poner los productos en el domicilio del consumidor en el menor tiempo posible (Srnicek, 2018). Trabajo que es organizado a destajo y vigilado por un amplio conjunto de aplicaciones informáticas soportadas en diversos dispositivos, que se constituyen como una red de vigilancia electrónica, desde los compradores a los manipuladores y empaquetadores 0 , desde luego, a los repartidores (ver por ejemplo Veen, Barratt y Goods, 2020; Revilla y Blázquez, 2021).

El comercio electrónico programa, así, una des-socialización del acto de compra para encerrar al consumidor digital en un circuito de referencias y representaciones, creado por una fórmula algorítmica mantenida a base de las propias informaciones y datos que ha dejado en sus navegaciones virtuales. Esto genera, en la mayor parte de los casos, la creación de auténticas "cámaras de resonancia" o "burbujas", en las que el consumidor se enfrenta a un universo autorreferencial que le pone en contacto con experiencias propias de su estrato social y cultural (Wright, 2015; Cohn, 2019), separándose de otras distintas y generando una tendencia general hacia la polarización que forma actualmente una de las preocupaciones relevantes en la sociología contemporánea (Heikkilä, Leguina y Purhonen, 2020). El círculo de referencias virtuales estrecha e individualiza al máximo la experiencia adquisitiva, haciendo invisibles todos los elementos humanos que no están directamente relacionados con la imagen ideal de la mercancía virtualizada y el deseo del consumidor previamente espiado, identificado, clasificado y seducido por su huella digital -las búsquedas previas, las compras anteriores, las visitas efectuadas a sitios web, las interacciones que realiza en todas las redes sociales y canales que frecuenta, los mensajes que deja en los foros- y que, finalmente los algoritmos procesan y le devuelven reflexivamente (y de forma también distorsionada), creándole su yo digital como su único yo posible, un yo consumidor y consumible (Chabault, 2019).

\section{EL CONSUMIDOR SOLITARIO}

Esta idea de comprar a solas -de un comprador interconectado, pero no humanamente comunicado-, impulsada por la ideología del tecnoconsumo, introduce en el ámbito del consumo una lógica paralela y necesaria: la del trabajar a solas, vieja utopía capitalista, por cierto, de la desestructuración de todos los elementos colectivos (y solidarios) de la división del trabajo social y, con ello, de la inutilización política del concepto de fuerza de trabajo que se ha perseguido desde los primeros grandes discursos tecnológicos de la tecnocracia liberal (Linhart, 2009). La idea de individualizar y descorporizar todo lo social que hay en lo económico en forma de máquinas, robots, teletrabajo, teleconsumo, autovigilancia y empresarialización del yo, ha sido y sigue siendo el ideal liberal capitalista de la construcción tecnológica de un homo economicus total, que se presenta como el preferente racional puro, ahora potenciado por la fuerza de los algoritmos y las redes. Pero detrás de este supuesto homo economicus electrónico lo que se oculta en realidad es un consumidor espiado, rastreado y construido por la inteligencia algorítmica y los medios de inducción y formación matemática de la demanda (ó Neil, 2017), a la vez que un ingente y creciente ejército de malos empleos localizados en el sector servicios, desde la logística hasta el reparto, pasando por la telepromoción, el seguimiento informático de los procesos o la clasificación de mercancías. Trabajos todos realizados a 
ritmos forzados, y donde se combinan las más altas tecnologías de la información para el control del desempeño con las más precarias y autoritarias formas de organización del trabajo: sin derechos, sin horarios, a plena disponibilidad y en muchas ocasiones poniendo los medios personales para realizar la actividad laboral (bicicletas, furgonetas, equipos electrónicos, redes familiares o personales, etc.).

La aceleración del ciberconsumo es una de los potenciadores esenciales del incremento de los malos empleos e introduce, de manera forzada, formas de trabajo indisolublemente ligadas a un tipo de economía donde producción, distribución y venta rompen el concepto de relaciones normalizadas y estabilizadas pera establecerlas sobre una inestabilidad y volatilidad radical. Los compromisos de rapidez o inmediatez en la entrega son esenciales para construir la imagen de un consumidor omnipotente y soberano, que es servido cumplidamente y al instante con sólo mostrar su deseo en el canal de la gran plataforma (Alonso, Fernández Rodríguez e Ibáñez Rojo, 2020). De este modo, se genera una presión inmensa sobre los empleados de este sector los cuales, dirigidos a través de una suerte de management algorítmico, o algocracia (Galiere, 2020) que les lleva a experimentar auténticas situaciones de temor y frustración ante la irregular carga de trabajo (a veces muy intensa, pero, y tal como nos cuenta Crouch (2019), muy escasa en algunos trabajos si no se está disponible full time) y los estrictos plazos de realización de las tareas, organizados en un exigente just-in-time (ver Huws, Spencer y Syrdal, 2018; Kellogg, Valentine y Christin, 2020). De este modo, la promesa de la "muerte de la distancia" termina generando un notable estrés entre una fuerza de trabajo precaria que se ve obligada a adaptarse a un escenario de entrega a demanda con enormes constricciones, y ante el que además es difícil reclamar al depender de un algoritmo del que nada se sabe.

Esta construcción de un habitus de consumidor instantáneo y a distancia (que no puede esperar, ni posponer su deseo y al que se le debe servir el objeto en su propio domicilio) aceptado y naturalizado como el estándar del consumidor actual, arrastra a los canales de distribución a una subasta a la baja de los derechos laborales y de los márgenes comerciales que le corresponden a los eslabonas más débiles de las cadenas de valor. Acelerando las entregas y construyendo como una necesidad inherente al e-commerce la rapidez o incluso la inmediatez, se presiona y saca de la competencia a los distribuidores más pequeños o al comercio tradicional que, ya sea de forma presencial, o en sus limitadas extensiones virtuales, tratan de seguir su actividad (Carrión, 2019). Del mismo modo, liberar nominalmente o contractualizar preferencialmente con el consumidor los costes de transporte y entrega de los productos, como estrategia de las grandes plataformas para fidelizar y hacer más competitiva su oferta -aumentando sus márgenes en todos los segmentos del negocio debido a su poder sobre el canal y el volumen de negocio-, va más allá de ser una oferta comercial o una forma de promoción: es una forma de demostración de dominio sobre el mercado, tanto en lo referente a su posición frente a los posibles competidores comerciales, como sobre los mercados de trabajo que generan, organizados estos en forma de subcontratación, externalización y subordinación de todas las formas posibles de empleo de mala calidad. El consumidor digital es el tipo de agente social perfecto para justificar la utilización de formas de trabajo donde la contractualización de servicios (muchas veces degradados o informales) se convierten en el estatuto de empleo considerado como imprescindible para ofrecer los servicios rápidos y competitivos que supuestamente exige el comprador que ha realizado su adquisición vía internet (Schor, Attwood-Charles, 2017; Huws, Spencer y Syrdal, 2018).

El comercio digital pasa a ser uno de los elementos fundamentales de la vida en la pantalla, según el ya clásico y clarividente diagnóstico de Sherry Turkle (1997): esto es, la formación de un consumidor electrónicamente aislado de todo acontecer no pasado por la realidad virtual. Mucho más que en épocas anteriores, el consumidor se va a ver fijado a un sistema de representaciones, signos y símbolos que han desplegado las plataformas de venta y que se entrelazan con la exposición de su imagen personal en las redes sociales (Instagram, Facebook, Twitter, etc.) o en los videojuegos online, donde el afán de exhibición idealizada y aumentada del narcisismo del yo informático 
se expresa mediante todo tipo de imágenes y selfies expuestas a modo de búsqueda de reconocimiento en la red, en la que la interacción servirá para construir ese "yo que deseamos ser". Esas representaciones, a su vez, retroalimentan de datos e información a la inteligencia artificial algorítmica de las plataformas comerciales. El consumidor virtual se conecta con sistemas de aceptación y refuerzo de su imagen, que lo encierran en un círculo digital diseñado para lograr su distanciamiento con respecto a cualquier espacio de lo social que signifique una conectividad interpersonal directa o una intersubjetividad no mediada por la tecnología (y su inevitable dimensión mercantil).

El productivismo de las formas dominantes de la compra digital hacen de la actividad adquisitiva del consumidor en internet una especie de trabajo de consumo (Dujarier, 2014): un inmenso gasto de tiempo, atención y sumisión que le obliga a seleccionar ofertas, actualizar tecnología, seguir vídeos formativos, solucionar problemas, gestionar diversas formas de pago, desplegar un número cada vez más ingente de aplicaciones, y todo ello simplemente para poder realizar la función básica de la compra (Patino, 2019; Wu, 2020)). Nos encontramos entonces ante un consumidor máximamente activado por las normas tecnológicas de las plataformas (y en general del mundo electrónico) y los sistemas de pago exigidos por las finanzas electrónicas (claves, números de tarjeta, más aplicaciones exclusivas para encuadrar los cobros, etc.). La idea del prosumidor tan ingenuamente defendida por la llamada economía colaborativa -moda cultural vestida de solidaria en su primera presentación en el contexto de la gran crisis financiera de este siglo, y luego inmediatamente engullida por la mercantilización más absoluta, perdiendo rápidamente su relación con el ámbito de lo público (Alonso, 2017)- toma, en la práctica del consumidor de plataformas, la virtualidad de ser el responsable de la autoproducción de una buena parte de las condiciones de realización de sus prácticas adquisitivas: programación técnica de los objetos, aprendizaje de las formas de uso, puesta al día del sistema tecnológico, relación con las extensiones comerciales, aplicaciones y actualizaciones de las marcas, conexión con otros consumidores en red, seguimiento de las entregas, acabados y montajes de las mercancías, y un largo etcétera de obligaciones disciplinarias imposibles de soslayar si se quiere mantener ese estilo de vida.

Tiempo y esfuerzo dedicado a la autoproducción de un conjunto de servicios finales que son asumidos y realizados por un consumidor al que se le pone a trabajar en su propio consumo, bajo el discurso (y la amenaza) de no quedar aislado, desenganchado y socialmente obsoleto. El canal en las economías de la plataforma impone todas las normas (tanto de la producción como de la distribución y el consumo) que pasan en primer lugar por activar al comprador, exigiéndole una predisposición absoluta y una aceptación total hacia todas las condiciones de uso que imponen las mediaciones informáticas en su proceso de producción telemático del deseo (y de su frustración).

\section{DISCUSIÓN FINAL}

El e-commerce se ha desarrollado entonces a través de las economías de plataforma como una auténtica ontología empresarial del consumo -como diría Mark Fisher (2016)-, que remata el proyecto neoliberal de construir todo el ser humano como un producto empresarial. Por tanto, el trabajo asociado a ese consumo a través de internet sólo tiene sentido en cuanto que sirve de la manera más directa y descarnada al beneficio empresarial de las plataformas, y no, como en los clásicos del reformismo social - por ejemplo, Durkheim-, a ningún tipo de solidaridad colectiva o proyecto común. La gig economy es una forma de capitalismo en el que los grandes distribuidores comerciales a través de plataformas informáticas o los gerentes de redes de intermediación de servicios fuerzan a que los recursos (capital y trabajo) lo pongan otros agentes implicados en el circuito económico, desde los fondos de inversión transnacionales a los propios trabajadores infra y subcontratados. Estos últimos tienen que poner sus medios personales (bicicletas, motocicletas, vehículos de carga, equipos informáticos, hogares, relaciones personales) para, simplemente, poder pujar por entrar en el juego de 
la distribución a domicilio, del transporte a personas, de la información y venta en los call centers o en la vigilancia y control de las demandas de las propias redes. Trabajos desmigajados y aislados, siempre conectados por aplicaciones informáticas que, además de controlar y disciplinar el desempeño de los subcontratados, disuelven, hasta anular, el sentido colectivo de las peculiares relaciones salariales estructuradas por un sistema de algoritmos despersonalizados que aíslan y despojan de control -y de derechos socialesa todo aquel que tiene algún contacto subordinado a él (Abdelnour y Mèda, 2019).

Lejos de construir una mitología del algoritmo y la robotización -en su maldad o su bondad como hoy vemos en miles de discursos al uso y abuso-, el sentido social de todas las formas de la economía de plataformas hay que buscarlo en su relación con un nuevo despliegue de la tecnología capitalista para extraer información, planificar estrategias, condicionar comportamientos y desposeer a los competidores ( $\mathrm{y}$ a los trabajadores) de sus recursos y sus poderes (Srnicek, 2018). Por ello, los algoritmos predictivos ponen en juego formas de vigilancia, adicción y programación en el mundo del consumo que, necesariamente, se combinan con formas de vigilancia, control y desestructuración social en el mundo del trabajo (Bloom, 2019; Zuboff, 2020). Y aunque este proyecto empresarial tiene sus límites -ya que, pese a la tendencia a buscar ese consumidor individualizado perfecto, el Big Data no siempre funcionará, pues nunca será lo suficientemente exhaustivo-, lo cierto es que la tendencia en el mercado parece imparable. Las plataformas arrastran a todas las empresas a tener que acudir a su entorno de negocio si simplemente quieren sobrevivir, y ello les otorga el dominio en la creación de sus propias reglas del mercado: un mercado cada vez más difícil de regular o defender institucionalmente, ya sea a nivel estatal o supranacional. El consumo organizado a partir de las empresas Big Tech ha creado y reforzado (y con las medidas preventivas y de confinamiento de la pandemia en grado máximo) una nueva clase de consumidores recluidos y socialmente desintegrados, que tiene dificultades incluso para representarse y percibirse a sí mismo como grupo social; las grandes plataformas de todo tipo utilizan sus datos recopilados para ofrecer un catálogo ingente de bienes y servicios a un ritmo imposible de procesar con cierta reflexión o detenimiento, por lo que el reclamo forzado de la atención y la sobreoferta -hasta el atracón real o imaginario- de posibilidades adquisitivas se convierte en el modo básico de relación con el cliente en la pantalla. Todo es excesivo y acelerado, y todo se construye a la medida de la información que el comprador ha ido facilitando en su experiencia adquisitiva previa, su ocio o, simplemente, sus consultas en las redes.

La customización de la oferta -algo así como tienes lo que quieras a la hora que quieras y servido en tu propia puerta si la mercancía es física- crea una imagen distorsionada pero extraordinariamente verosímil de máxima personalización y dominio del mundo de las redes por parte del comprador, donde desaparece todo lo que no se procesa por esta vía, y, por tanto, las mediaciones ideológicas y mercantiles que crea este tipo de oferta en las plataformas. La idea misma de conciencia colectiva como fundamento de la sociabilidad -de nuevo Durkheim- se desvanece en un yo profundamente asocial, y por ello ciego para observar los tipos y usos de trabajo que ese consumo de plataforma conlleva. Un trabajo que tiende a desintegrarse y aislarse con la misma lógica tecnológica que el consumo que lo reclama y que, extrañamente, combina las formas tecnológicas más sofisticadas en su gestión y vigilancia con modos despóticos y generadores de gran precariedad en su planteamiento de las relaciones laborales. Estaríamos en una especie de nueva Edad Media laboral -por utilizar la brillante caracterización que Umberto Eco (1974) hacía de los peligros de nuestra época-, donde la máxima innovación tecnológica crea y mantiene colectivos cuyo estatuto salarial -muchas veces discutido y reivindicado jurídicamente- siempre se reproduce en la alegalidad más flagrante, lo que hace que, más que postmodernas, estas formas de empleo creadas por el capitalismo de plataformas sean directamente antimodernas y nos retrotraigan a formas de vida y modos de exclusión y vulnerabilidad social que pensábamos que habíamos superado con los procesos de modernización social del siglo pasado. Paradójicamente, el modelo cuenta, en muchas ocasiones, con el apoyo tácito de los consumidores que, como señalan Culperer y Thelen 
(2020), se opone en muchos casos a cualquier tipo de regulación de la actividad de estas plataformas que pueda perjudicar la flexibilidad del proceso de consumo. Esto debe hacernos reflexionar sobre la responsabilidad del consumidor, aunque generalmente este no deja de ser un consumidor en muchos casos precario, cuya nueva demanda es la inmediatez ante sus horarios desestructurados.

La economía de plataformas, por concluir, está provocando cambios extraordinarios en las economías contemporáneas y, por ende, en nuestras sociedades. La digitalización de los procesos de trabajo y consumo parece de momento imparable, y las empresas han encontrado en el uso de los algoritmos una estrategia de gestión extraordinaria, tanto de los procesos de organización del flujo de trabajo como de la segmentación del consumidor. El algoritmo, hoy, mueve al consumo. El hiperconsumidor solitario contemporáneo satisface su deseo recurriendo a la plataforma, donde se le devuelve, de modo especular, una imagen de sí mismo en la que puede soñar con la libertad de elección, consiguiendo prácticamente cualquier cosa a través de la red, una vez que la oferta ha sido convenientemente ajustada en precios ante la extensión del low cost a prácticamente todos los productos y servicios (Alonso, Fernández Rodríguez e Ibáñez Rojo, 2020). Y frente a una cierta ambivalencia de los discursos sobre la desaparición del trabajo, en el que supuestamente este perderá su rol frente al ascenso de la inteligencia artificial (ver sobre estos discursos Lahera Sánchez, 2019; Lobera, Fernández Rodríguez y Torres-Albero, 2020), al final no deja de ser el trabajo precario el que gestiona la producción y logística de dicho catálogo. Y no parece existir alternativa al modelo: si bien la reciente pandemia nos ha mostrado una movilización social en favor del consumo local y de proximidad, culturalmente, este modelo de consumo se impone como legítimo, racional y razonable, dejando pocos espacios para la disidencia. En todo caso, no podemos comprender el éxito de la economía de plataformas y la extensión de sus nuevas formas de empleo sin centrar nuestra mirada en el modelo de consumo digital que está, realmente, alimentando esta profundización en la organización postfordista de la producción. Por ello, consideramos que es imprescindible enfatizar la importancia de analizar ese vínculo entre trabajo y consumo -muchas veces velado, oscurecidocuando exploramos este nuevo escenario de la economía de plataformas, señalando el rol que el consumo juega a la hora de impulsar esta forma novedosa de organización del capitalismo contemporáneo.

\section{FINANCIACIÓN}

Este trabajo ha sido financiado en el contexto del proyecto de investigación del Ministerio de Ciencia, Innovación y Universidades, con referencia PGC2018-097200-B-I00.

\section{REFERENCIAS}

Abdelnour, S. y Méda, D. (Eds.) (2019). Les nouveaux travailleurs des aplis. París: Presses Universitaires de France.

Acquier, A., Daudigeos, T. y Pinkse, J. (2017). Promises and paradoxes of the sharing economy: An organizing framework. Technological Forecasting \& Social Change, (125), 1-10. http:// dx.doi.org/10.1016/j.techfore.2017.07.006

Alonso, L. E. (2017). Consumo colaborativo: Las razones de un debate. Revista Española de Sociología, 26(1), 87-95. http://doi:10.22325/fes/res.2017.4 
Alonso, L. E., Fernández Rodríguez, C. J. e Ibáñez Rojo, R. (2020). Del low cost a la gig economy: el consumo en el postfordismo del siglo XXI. En Alonso, Luis E., Fernández Rodríguez, C. J. e Ibáñez Rojo, R. (Eds.), Estudios sociales sobre el consumo (pp.241-260). Madrid: Centro de Investigaciones Sociológicas.

Álvarez Hernández, G. y Pérez Zapata, O. (2020). Hacia la platamorfización: el caso de una plataforma digital cualificada. En Riesco-Sanz, A. (Ed.), Fronteras del trabajo asalariado (pp. 155-184). Madrid: La Catarata.

Aroles, J., Mitev N. y de Vaujany, F.-X. (2019). Mapping themes in the study of new work practices. New Technology, Work and Employment, 34(3), 265-299. https://doi.org/10.1111/ NTWE.12146

Beer, D. (2009). Power through the algorithm? Participatory web cultures and the technological unconscious. New Media \& Society, 11(6), 985-1002. https://doi. org/10.1177/1461444809336551

Bloom, P. (2019). Monitored: Business and Surveillance in a Time of Big Data. Londres: Pluto.

Carrión, J. (2019). Contra Amazon. Barcelona: Galaxia Gutemberg.

Cohn, J. (2019). The Burden of Choice: Recommendations, Subversion, and Algorithmic Culture. New Brunswick, NJ: Rutgers University Press.

Crouch, C. (2019). Will the Gig Economy Prevail? Cambridge, Polity.

Culperer, P. y Thelen, K. (2020). Are We All Amazon Primed? Consumers and the Politics of Platform Power. Comparative Political Studies, 53(2), 288-318. https://doi. org/10.1177/0010414019852687

Dujarier, M-A. (2014). Le travail du consommateur, De Mac Do à eBay : comment nous coproduisons ce que nous achetons. París: La Découverte.

Chabault, V. (2020). Éloge du magasin. Contre l'amazonisation. París: Gallimard.

Eco, U. (1974). La Edad media ha comenzado ya. En Eco, U. et al, La nueva Edad Media, (pp. 9-36). Madrid: Alianza.

Fin, E. (2018). La búsqueda del algoritmo. Imaginación en la era de la informática. Barcelona: Alpha Decay.

Fisher, M. (2016). Realismo capitalista. ¿No hay alternativa? Buenos Aires, Caja Negra.

Fleming, P. (2017). The Human Capital Hoax: Work, Debt and Insecurity in the Era of Uberization. Organization Studies, 38(5), 691-709. https://doi.org/10.1177/0170840616686129

Fourcade, M. y Healy, K. (2017). Seeing like a Market. Socio-Economic Review, 15(1): 9-29. https://doi.org/10.1093/ser/mww033

Frick, V., Matthies, E., Thøgersen, J. y Santarius, T. (2020). Do online environments promote sufficiency or overconsumption? Online advertisement and social media effects on clothing, digital devices, and air travel consumption. Journal of Consumer Behaviour, 20(2), 288-308. https://doi.org/10.1002/cb.1855

Galiere, S. (2020). When food-delivery platform workers consent to algorithmic management: a Foucauldian perspective. New Technology, Work and Employment, 35(3), 357-390. https:/ / doi.org/10.1111/ntwe.12177

Gillespie, T. (2014). The Relevance of Algorithms. En Gillespie, T., Boczkowski, P. J., Foot, K. A. (Eds.), Media Technologies: Essays on Communication, Materiality, and Society (pp. 167193). Cambridge, MA: MIT Press. 
Heikkilä, R., Leguina, A. y Purhonen, S. (2020). The stratification of media usage in Finland, 2007-2018: Signs of socio-political polarization? New Media and Society. https://doi. org/10.1177/1461444820971612

Howcroft, D. y Bergvall-Kåreborn, B. (2019). A Typology of Crowdwork Platforms. Work, Employment and Society, 33(1), 21-38. https://doi.org/10.1177/0950017018760136

Huws, U., Spencer, N. H. y Syrdal, D. S. (2018). Online, on call: the spread of digitally organised just-in-time working and its implications for standard employment models. New Technology, Work and Employment, 33(2), 113-129. https://doi.org/10.1111/ntwe.12111

Kellogg, K.C., Valentine, M.A. y Christin, A. (2020). Algorithms at Work: The New Contested Terrain of Control. Academy of Management Annals, 14(1), 366-410. https://doi.org/10.5465/ annals.2018.0174

Lahera Sánchez, A. (2019). Digitalización, robotización, trabajo y vida: cartografías, debates y prácticas. Cuadernos De Relaciones Laborales, 37(2), 249-273. https://doi.org/10.5209/ crla.66037

Lash, S. (2007). Power after Hegemony: Cultural Studies in Mutation. Theory, Culture \& Society 24(3), 55-78. https://doi.org/10.1177/0263276407075956

Linhart, D. (2009). Travailler sans les autres ? París: Seuil.

Lipovetsky, G. (2020). Gustar y emocionar. Ensayo sobre la sociedad de la seducción. Barcelona: Anagrama.

Lobera, J., Fernández Rodríguez, C. J. y Torres-Albero, C. (2020). Privacy, Values and Machines: Predicting Opposition to Artificial Intelligence. Communication Studies, 71(3), 448-465. https://doi.org/10.1080/10510974.2020.1736114

O’Neil, C. (2017). Armas de destrucción matemática, Madrid, Capitán Swing.

Parguel, B., Lunardo, R. y Benoit-Moureau, F. (2017). Sustainability of the sharing economy in question: When second-hand peer-to-peer platforms stimulate indulgent consumption. Technological Forecasting \& Social Change, 125, 48-57. http://dx.doi.org/10.1016/j. techfore.2017.03.029

Patino, B. (2019). La civilisation du poisson rouge : Petit traité sur le marché de l'attention. París: Grasset.

Ravanelle, A. (2019). Precariedad y pérdida de derechos. Historias de la economía gig. Madrid: Alianza.

Revilla, J. C. y Blázquez Martín, V. (2021). Uneasy riders: contradictorias lógicas disciplinarias para una posición laboral imposible. Revista Española de Sociología, 30(2): a35. https:/ / doi.org/10.22325/fes/res.2021.35

Rosenblat, A. y Stark, L. (2016). Algorithmic labor and information asymmetries: a case study of Uber's drivers. International Journal of Communication, 10, 3758-3784. https://ijoc.org/ index.php/ijoc/article/view/4892/1739

Sandin, É. (2016). La siliconisation du monde. L'irrésistible expansion du libéralisme numérique. París: L’Echappée.

Schor, J. y Attwood-Charles, W. (2017). The 'Sharing economy': labour inequality and socialconnection on for-Profit Platforms. Sociology Compass, 11(8), 175-183. https://doi. org/10.1111/soc4.12493 
Seaver, N. (2017). Algorithms as culture: Some tactics for the ethnography of algorithmic systems. Big Data \& Society https://doi.org/10.1177/2053951717738104

Shapiro, A. (2020). Dynamic exploits: calculative asymmetries in the on-demand economy. New Technology, Work and Employment, 35(2), 162-177. https://doi.org/10.1111/ntwe.12160

Srnicek, N. (2018). Capitalismo de plataformas. Buenos Aires: Caja Negra.

Striphas, T. (2015). Algorithmic culture. European Journal of Cultural Studies, 18(4-5), 395-412. https://doi.org/10.1177/1367549415577392

Tegmark, M. (2018). Vida 3.0. qué significa ser humano en la era de la inteligencia artificial. Barcelona: Taurus.

Tolentino, J. (2019). Trick Mirror. Reflections on Self-Delusion. Nueva York: Random House.

Turkle, S. (1997). La vida en la pantalla. La construcción de la identidad en la era de internet. Barcelona: Paidós.

Veen, A., Barratt T. y Goods, C. (2020). Platform-Capital's 'App-etite' for Control: A Labour Process Analysis of Food-Delivery Work in Australia. Work, Employment and Society, 34(3) 388-406. https://doi.org/10.1177/0950017019836911

Wood, A. J., Graham, M., Lehdonvirta, V., y Hjorth, I. (2019). Good Gig, Bad Gig: Autonomy and Algorithmic Control in the Global Gig Economy. Work, Employment and Society, 33(1) 56-75. https://doi.org/10.1177/0950017018785616

Wright, D. (2015). Understanding Cultural Taste: Sensation, Skill and Sensibility. New York: Palgrave MacMillan.

Wu, T. (2020). Comerciantes de atención. Madrid: Capitán Swing.

Zuboff, S. (2020). La era del capitalismo de la vigilancia. Barcelona: Paidós. 